\title{
Ultra-rare mutations in SRCAP segregate in Caribbean Hispanic families with Alzheimer disease
}

\section{OPEN}

Badri N. Vardarajan, PhD Giuseppe Tosto, MD,

$\mathrm{PhD}$

Roger Lefort, $\mathrm{PhD}$

Lei $\mathrm{Yu}, \mathrm{PhD}$

David A. Bennett, MD

Philip L. De Jager, MD, $\mathrm{PhD}$

Sandra Barral, PhD

Dolly Reyes-Dumeyer, BS

Peter L. Nagy, MD, PhD

Joseph H. Lee, PhD

Rong Cheng, $\mathrm{PhD}$

Martin Medrano, MD

Rafael Lantigua, MD

Ekaterina Rogaeva, PhD

Peter St George-Hyslop,

MD

Richard Mayeux, MD

Correspondence to

Dr. Mayeux:

rpm2@cumc.columbia.edu

Supplemental data at Neurology.org/ng

\section{ABSTRACT}

Objective: To identify rare coding variants segregating with late-onset Alzheimer disease (LOAD) in Caribbean Hispanic families

Methods: Whole-exome sequencing (WES) was completed in 110 individuals from 31 Caribbean Hispanic families without APOE $\& 4$ homozygous carriers. Rare coding mutations segregating in families were subsequently genotyped in additional families and in an independent cohort of Caribbean Hispanic patients and controls. SRCAP messenger RNA (mRNA) expression was assessed in whole blood from mutation carriers with LOAD, noncarriers with LOAD, and healthy elderly controls, and also from autopsied brains in 2 clinical neuropathologic cohort studies of aging and dementia.

Results: Ten ultra-rare missense mutations in the Snf2-related CREBBP, activator protein (SRCAP), were found in 12 unrelated families. Compared with the frequency in Caribbean Hispanic controls and the Latino population in the Exome Aggregation Consortium, the frequency of SRCAP mutations among Caribbean Hispanic patients with LOAD was significantly enriched $(p=$ 1.19e-16). mRNA expression of SRCAP in whole blood was significantly lower in mutation carriers with LOAD, while the expression in whole blood and in the brain was significantly higher in nonmutation carriers with LOAD. Brain expression also correlated with clinical and neuropathologic endophenotypes.

Conclusions: WES in Caribbean Hispanic families with LOAD revealed ultra-rare missense mutations in SRCAP, a gene expressed in the brain and mutated in Floating-Harbor syndrome. SRCAP is a potent coactivator of the CREB-binding protein and a regulator of DNA damage response involving ATP-dependent chromatin remodeling. We hypothesize that increased expression in LOAD suggests a compensatory mechanism altered in mutation carriers. Neurol Genet 2017;3: e178; doi: 10.1212/NXG.0000000000000178

\section{GLOSSARY}

CBP = CREB-binding protein; $\mathbf{C l}=$ confidence interval; DLPFC $=$ dorsolateral prefrontal cortex; ExAC = Exome Aggregation Consortium; FPKM = fragments per kilobase per million fragments mapped; GEE = generalized estimating equation; IRB = institutional review board; $\mathbf{L O A D}=$ late-onset Alzheimer disease; $\mathbf{M C l}=$ mild cognitive impairment; $\mathbf{m R N A}=$ messenger RNA; OR = odds ratio; QC = quality control; SNP = single nucleotide polymorphism; SNV = single nucleotide variant; $\mathbf{W E S}$ = whole-exome sequencing.

Progress has been made in understanding the genetics of late-onset Alzheimer disease (LOAD), ${ }^{1,2}$ but gaps in its genetic influence still need investigation. Common variants play a role in disease risk, but functionally important rare or ultra-rare variants may help to explain the remaining heritability ${ }^{2,3}$ undetected by genome-wide association studies. Sequencing of large families multiply affected by LOAD increases the ability to detect novel variants conferring risk.

\footnotetext{
From the Taub Institute for Research on Alzheimer's Disease and the Aging Brain (B.N.V., G.T., R. Lefort, P.L.D.J., S.B., D.R.-D., J.H.L., R.C., R. Lantigua, R.M.); Gertrude H. Sergievsky Center (B.N.V., G.T., S.B., D.R.-D., J.H.L., R.C., R.M.); Department of Neurology (P.L.D.J., S.B., R. M.), Department of Psychiatry (R.M.), Department of Systems Biology (B.N.V.), Department of Medicine (R. Lantigua), and Department of Pathology and Cell Biology (R. Lefort, P.L.N.), College of Physicians and Surgeons, Columbia University, New York Presbyterian Hospital; Department of Epidemiology (J.H.L., R.M.), School of Public Health, Columbia University, New York; Tanz Centre for Research in Neurodegenerative Diseases (E.R., P.S.G.-H.) and Department of Medicine (E.R., P.S.G.-H.), University of Toronto, Krembil Discovery Tower, ON, Canada; Department of Clinical Neurosciences (P.S.G.-H.), Cambridge Institute for Medical Research, University of Cambridge, UK; Rush Alzheimer's Disease Center (L.Y., D.A.B.), Rush University Medical Center, Chicago, IL; Program in Medical and Population Genetics (P.L.D.J.), Broad Institute, Cambridge, MA; and School of Medicine (M.M.), Mother and Teacher Pontifical Catholic University, Santiago, Dominican Republic.

Funding information and disclosures are provided at the end of the article. Go to Neurology.org/ng for full disclosure forms. The Article Processing Charge was funded by the NIH.

This is an open access article distributed under the terms of the Creative Commons Attribution-NonCommercial-NoDerivatives License 4.0 (CC BY-NC-ND), which permits downloading and sharing the work provided it is properly cited. The work cannot be changed in any way or used commercially without permission from the journal.
} 
The frequency of LOAD among Caribbean Hispanic multiplex families from the Dominican Republic was 5 times higher than expected for similarly aged individuals in a non-Hispanic white population from the United States, ${ }^{4}$ and inbreeding was a significant predictor of LOAD in this population after adjusting for $A P O E-\varepsilon 4$ genotype, an established genetic risk factor. ${ }^{5}$

To identify novel variants associated with the risk of LOAD, we conducted wholeexome sequencing in 31 Caribbean Hispanic families (table e-1 at Neurology.org/ng) with 4 or more affected individuals, no mutations in known $\mathrm{AD}$ genes, specifically PSEN1, PSEN2, or APP, and no APOE $\varepsilon 4$ homozygotes. For each family, we sequenced at least 2 affected and 1 unaffected member aged 65 years or older.

METHODS Sample selection. Families were recruited as a part of a 15-year family-based study with institutional review board (IRB) approval based in the Dominican Republic. ${ }^{6}$ Thirtyone families (98 affected and 12 unaffected individuals) were selected for sequencing (mean age at onset was 74.8 wa8.3 years, and $63.1 \%$ were women) (table e- $1, \mathrm{a}-\mathrm{c}$ ). All family members had standard neuropsychological tests and neurologic examinations to verify their clinical status and for diagnoses based on NINCDSADRDA criteria. ${ }^{7,8}$

Postmortem human brain samples. Data were obtained from 2 clinical neuropathologic cohort studies: the ROS 9 and the MAP. ${ }^{10}$ The IRB of Rush University Medical Center previously approved both studies. Clinical evaluations were used to determine NINCDS-ADRDA ${ }^{7,8}$ criteria for dementia annually. ${ }^{11-13}$ At death, a clinical diagnosis opinion was provided by a neurologist. ${ }^{14}$ Neuropathologic evaluations included neuritic plaques, diffuse plaques, and neurofibrillary tangles in 5 cortical regions, scaled and averaged to obtain a composite score. ${ }^{15}$ Participants who met intermediate or high likelihood were rendered pathologic diagnosis of LOAD. ${ }^{16,17}$

WHOLE-EXOME SEQUENCING Sample preparation. Qiagen's Gentra Puregene and FlexiGene kits were used to extract high-molecular-weight DNA from fresh or frozen $\left(<-80^{\circ} \mathrm{C}\right)$ samples. DNA from saliva was isolated using prepIT.L2P (DNA Genoteck Inc., Ottawa, ON, Canada). Cell lines from lymphocytes (in 13 probands) were used when high-quality blood DNA was not available. The concentration of DNA was determined using a NanoDrop spectrophotometer.

Sequencing. The Illumina TruSeq DNA preparation kit was used to prepare and index genomic DNA libraries. Custom oligonucleotide baits in the TruSeq Exome Enrichment kit were used to capture coding regions and splice sites and amplified according to the Illumina protocol. The DNA samples were multiplexed in batches of 12 samples with index "barcode" primers. These were sequenced using the Illumina Genome Analyzer IIx, HiSeq 2000, and MiSeq platforms (illumina.com) as paired-end reads over 82-307 cycles. Demultiplexing by barcode retrieved individual samples from sequencing pools. We obtained a high coverage across the samples at an average depth of $>60 \times$ per sample.

Follow-up genotyping. Putative variants were confirmed and population frequencies estimated by genotyping the discovery samples, additional family members, and unrelated controls of the same ancestry (table e-1). Allele frequencies of novel variants were estimated from 1,949 unrelated patients and 318 healthy elderly controls similar in age and ancestry. ${ }^{6}$ Genotypes were generated using the KASP genotyping technology, which uses allele-specific PCR for accurate calling of single nucleotide polymorphisms (SNPs) and Indels. ${ }^{18}$

Analytical methods. Burrows-Wheeler Aligner ${ }^{19}$ was used to align sequence reads to the reference genome build 37. Sequencing data quality control (QC) was performed using the Genome Analysis Toolkit (GATK), ${ }^{20}$ followed by variant calling using the UnifiedGenotyper and VariantRecalibrator modules. Variants that passed QC were annotated by ANNOVAR $^{21}$ that included functional prediction by SIFT $^{22}$ and PolyPhen. ${ }^{23}$

STATISTICAL METHODS Association tests. Variants that were validated by follow-up genotyping were tested for association with LOAD using generalized estimating equations (GEEs), which accounts for the familial correlation. We adjusted for age and sex using data from the families and unrelated healthy controls. We used GEE to conduct single variant and burden tests.

For joint analyses of multiple variants, we summed the number of rare variant alleles found in all individuals and tested association using GEE, adjusting for familial correlation, age and sex, and $A P O E \& 4$. The $p$ value threshold required to define statistical significance using a rare variant burden analysis for 20,000 genes would be $2.5 \times 10^{-6}$. However, this would be difficult to achieve by assessing rare or ultra-rare variants in a data set of this size. Thus, to determine whether or not the variants discovered were enriched in families with $\mathrm{AD}$ in subsequent analyses, we used data from the Exome Aggregation Consortium (ExAC) (exac.broadinstitute.org) combined with the Caribbean Hispanic controls.

Exome Aggregation Consortium. The ExAC database ${ }^{24}$ contains whole-exome data from 60,706 unrelated adults sequenced as part of various disease-specific (excluding $\mathrm{AD}$ ) and population genetic studies from 6 ethnic groups. For disease association analyses, we 
Figure 1 Workflow of the experiment and yield at each step

- WES multisample calling

- Variant recalibration and QC

200,000 high-quality variants

- Novel, coding, damaging SNVs present in 4 or more affected individuals in multiple families or segregating in 1 family

- Frameshift indels present in 2 or more families and ExAC frequency $<2 \%$

67 SNVs and 11 indels

- Follow-up genotyping in discovery cohort, additional family members and 498 controls

- Family-based association testing using GEE

22 variants significant at a Bonferroni corrected $p<6.3 \mathrm{E}-03$

- 4 genes with significant variants expressed in the brain

- 1 gene with 9 additional missense variants segregating in the discovery exome

10 missense SRCAP variants discovered in WES data

- Ten missense genotyped in 31 discovery families, additional 47
families and unrelated case-control sample of $\mathrm{CH}$ ancestry
- Joint burden of $S R C A P$ significant in the genotyped data
(OR=1.91, $p=0.016)$
Burden of $S R C A P$ mutations significant when jointly
compared with controls from $\mathrm{CH}$ population and ExAC
controls at $p=1.19 \mathrm{e}-16$, OR=2.54
- Compared SRCAP expression in blood from LOAD mutation
carriers and wild-type patients with LOAD and healthy individuals
SRCAP expression was significantly lower in affected
mutation carriers with LOAD than in the other two groups

$\mathrm{CH}=$ Caribbean Hispanic; ExAC = Exome Aggregation Consortium; GEE = generalized estimating equation; $\mathrm{OR}$ = odds ratio; $\mathrm{QC}$ = quality control; $\mathrm{SNV}$ = single nucleotide variant; WES $=$ whole-exome sequencing.

compared the allele frequencies of suspected variants in the healthy controls from the Caribbean Hispanic cohort to the Latino, Caucasian, and African subpopulations of the ExAC database using a Fisher exact test to avoid differences based on ancestry. Subsequent disease-associated analyses were only conducted when there were no statistically significant differences in allele frequency between the Caribbean Hispanic controls and the ExAC subpopulations. To confirm associations of genetic variants with LOAD, we compared the variant allele frequencies by selecting 1 patient from each Caribbean Hispanic family with both unaffected unrelated controls and ExAC combined using a Fisher exact test.

Gene expression in the postmortem human brain. RNASeq data came from the 541 ROS-MAP postmortem human brain samples (average age at death was 88.4 NA6.7 years; $63.0 \%$ were women and 97.4 selfidentified Caucasian ancestry). RNA was extracted from the gray matter of frozen dorsolateral prefrontal cortex tissue using the Qiagen miRNeasy mini kit (Cat. no. 217004) and the RNase-free DNase Set (Cat. no. 79254) and quantified using NanoDrop. RNA-Seq library was prepared on the Broad Institute's Genomics Platform using the strand-specific dUTP method ${ }^{25}$ with poly-A selection. ${ }^{26}$ Sequencing was performed on the Illumina HiSeq with $101 \mathrm{bp}$ paired-end reads and minimal coverage of $50 \mathrm{M}$ reads. Reads were aligned to the reference genome (GRCh37/hg19) using Bowtie and gene expression, measured as fragments per kilobase per million fragments mapped (FPKM), and estimated using RSEM software. Quantile normalization was applied to the FPKM calls, with batch effect removed using Combat. ${ }^{27}$ Logarithm base 2 transformation was applied to the gene expression level prior to the analyses.

The Student $t$ test compared brain expression levels between LOAD and controls. Regression models were used to examine the association of gene expression with LOAD and with level of cognition proximate to death. Regression models were used to examine the relation between gene expression and a postmortem diagnosis of LOAD or with LOAD pathology. All models were adjusted for age at death, sex, education, postmortem interval RNA integrity number, and $A P O E \varepsilon 4$ status. Analyses were performed using SAS software, version 9.3, of the SAS(R) system for Linux.

RESULTS Whole-exome sequencing. Variant calling, recalibration, and application of QC filters resulted in 290,623 single nucleotide variants (SNVs) and indels called across the 110 individuals from the 31 families (figure 1). The mean depth of sequence coverage was over 60 -fold across individuals. We prioritized damaging SNVs by SIFT ${ }^{22}$ or by PolyPhen ${ }^{28}$ and indels in 78 genes segregating with LOAD in families (figure e-1, A and B), which were confirmed by genotyping in the discovery families, additional family members (265 affected and 61 unaffected individuals), and a set of 318 unrelated controls of similar age and ancestry. Using GEE adjusted for age, sex, and $A P O E$ genotype, we found $22(28 \%)$ of the 78 selected variants (in 22 genes) significantly associated with LOAD ( $p=6.4 \mathrm{e}-03$ corrected for multiple testing) (table e-2). Only 4 of these 22 genes were known to be expressed in brain-ADCYG, CIT, SRCAP, and SVOPL. The variants in SRCAP, 
Table 1 Missense variants in SRCAP found in WES of Caribbean Hispanic families

\begin{tabular}{|c|c|c|c|c|c|c|c|c|c|c|c|c|c|}
\hline \multirow[b]{2}{*}{$\mathrm{Chr}$} & \multirow[b]{2}{*}{ Start } & \multirow[b]{2}{*}{ Gene } & \multirow[b]{2}{*}{ Ref } & \multirow[b]{2}{*}{ Obs } & \multirow[b]{2}{*}{ AA change ${ }^{a}$} & \multicolumn{2}{|c|}{ Discovery WES ${ }^{\mathbf{b}}$} & \multicolumn{2}{|c|}{$\begin{array}{l}\text { Genotyping in } \\
\text { discovery } \\
\text { families }^{c}\end{array}$} & \multicolumn{2}{|c|}{ Validation genotyping cohort } & \multicolumn{2}{|c|}{ ExAC frequencies } \\
\hline & & & & & & $\begin{array}{l}\text { AFF } \\
\text { samples }^{d}\end{array}$ & FAM $^{e}$ & $\begin{array}{l}\text { AFF } \\
\text { samples }^{d}\end{array}$ & FAM $^{\text {e }}$ & $\begin{array}{l}\text { Frequency } \\
\text { in familial } \\
\text { load cases }^{f}\end{array}$ & $\begin{array}{l}\text { Frequency } \\
\text { in elderly } \\
\text { unaffected }(>65 y)^{f}\end{array}$ & Latino & $\begin{array}{l}\text { Global } \\
\text { frequency }\end{array}$ \\
\hline 16 & 30731484 & SRCAP & G & A & R940Q & 1 & 1 & 1 & 1 & $4.30 e-04$ & $2.92 \mathrm{e}-03$ & $8.64 \mathrm{e}-05$ & $8.24 \mathrm{e}-06$ \\
\hline 16 & 30732143 & SRCAP & $\mathrm{T}$ & C & S1033P & 5 & 3 & 12 & 4 & $8.89 e-03$ & $5.85 e-03$ & $7.45 e-03$ & $7.41 \mathrm{e}-04$ \\
\hline 16 & 30732600 & SRCAP & G & A & $\mathrm{R} 1115 \mathrm{H}$ & 4 & 1 & 6 & 1 & $1.28 \mathrm{e}-03$ & 0 & 0 & $8.24 \mathrm{e}-06$ \\
\hline 16 & 30732644 & SRCAP & $\mathrm{C}$ & $\mathrm{T}$ & P1130s & 1 & 1 & 2 & 2 & $4.71 \mathrm{e}-03$ & $7.35 e-03$ & $2.94 \mathrm{e}-03$ & $2.57 e-03$ \\
\hline 16 & 30734934 & SRCAP & A & G & I1397V & 3 & 1 & 4 & 1 & $1.93 e-03$ & 0 & $0.00 e+00$ & $3.30 e-04$ \\
\hline 16 & 30748932 & SRCAP & C & G & S2524C & 2 & 1 & 1 & 1 & $1.27 \mathrm{e}-02$ & $2.96 \mathrm{e}-03$ & $1.30 \mathrm{e}-03$ & $2.04 \mathrm{e}-03$ \\
\hline
\end{tabular}

Abbreviations: ExAC $=$ Exome Aggregation Consortium; WES $=$ whole-exome sequencing.

${ }^{\text {a } A A}$ change: amino acid change caused by the mutation.

${ }^{b}$ Discovery WES: number of affected individuals and families identified as carrying an SRCAP variant in the WES experiment in 110 individuals (31 families).

${ }^{\mathrm{c}}$ Genotyping in discovery families: number of affected individuals and families identified as carrying an SRCAP variant in the validation genotyping in all the members of the 31 discovery families.

${ }^{\mathrm{d}}$ AFF samples: number of familial-affected samples carrying the SRCAP variant.

e FAM: number of families carrying the SRCAP variant.

${ }^{f}$ Frequency in the affected and unaffected individuals included familial and unrelated cases and controls.

$A D C Y 6$, and SVOPL were absent in unaffected Caribbean Hispanic individuals, but only SRCAP met criteria for further analyses by having multiple, putatively damaging variants segregating in more than 1 of the 31 families.

We observed 10 ultra-rare missense mutations, defined as having an allele frequency $\leq 0.5 \%$, in SRCAP in 12 families (table 1). Seven of these mutations were predicted to be damaging by either PolyPhen or SIFT and had a Combined Annotation Dependent Depletion ${ }^{29}>10$. Twenty-four $(24.4 \%)$ of the 98 affected individuals sequenced within the 31 families were carriers of SRCAP mutations. This was significantly higher than the expected frequency based on data from ExAC database ${ }^{24}$ (1.79 expected mutations; $p=1.38 \mathrm{e}-05$ using a binomial test).

All 10 SRCAP mutations were genotyped in additional members of the 31 families and segregated with affection status but with incomplete penetrance (figures e-3, A-D and e-4). Of the 265 patients with LOAD in the 31 families, 47 patients (17.67\%) carried at least 1 of the 10 missense mutations in SRCAP compared with 2 of the $61(3.2 \%)$ unaffected individuals from these families. Variant p.R1115H segregated with LOAD in the largest sibship in an age-dependent manner (supplementary methods, figure $e-2$, tables $e-3$, $a$ and $b$ ) and was absent in Caribbean Hispanic controls and ExAC individuals of European, Latino, and African ancestry. p.R1115H was also found within $0.35 \mathrm{MB}$ of a linkage peak (logarithm (base 10) of odds =1.8). A single patient was homozygous for an SRCAP p.S2524C mutation and had an earlier age at onset compared with the average age in the cohort (63 vs 73.8 years). In family 1755 (figure 2), there were 2 compound heterozygous carriers (p.L2919F and p.S1033P) with earlier ages at onset (55 and 59 years) compared with LOAD patients without $S R C A P$ mutations $(p=0.023)$ or patients who had a single $S R C A P$ mutation $(p=0.018)$. Of interest, no unaffected family member or unrelated control was a homozygous or compound heterozygous carrier.

A joint burden analysis within the 31 discovery families using GEE adjusted for familial correlation, age, sex, and $A P O E$ genotype found a 6-fold increase in the risk of LOAD (odds ratio $[\mathrm{OR}]=5.94 ; 95 \%$ confidence interval [CI] 1.59-22.27, $p=8.2 \mathrm{e}-03$ ). Adding the additional 47 families resulted in a 2.5fold risk of LOAD in families (OR $=2.54 ; 95 \% \mathrm{CI}$ $1.03-6.21, p=0.04)$. We then tested the joint burden of the 10 SRCAP variants in all the genotyped individuals in both sets of families, 1,949 unrelated LOAD cases and 318 unrelated elderly controls (table e-1b). The risk of LOAD associated with SRCAP remained increased $(\mathrm{OR}=1.92 ; 95 \%$ CI $1.13-$ 3.28, $p=0.016)$ after including $A P O E \varepsilon 4$ in the model $(\mathrm{OR}=1.82 ; 95 \%$ CI 1.04-3.19, $p=0.036)$. 


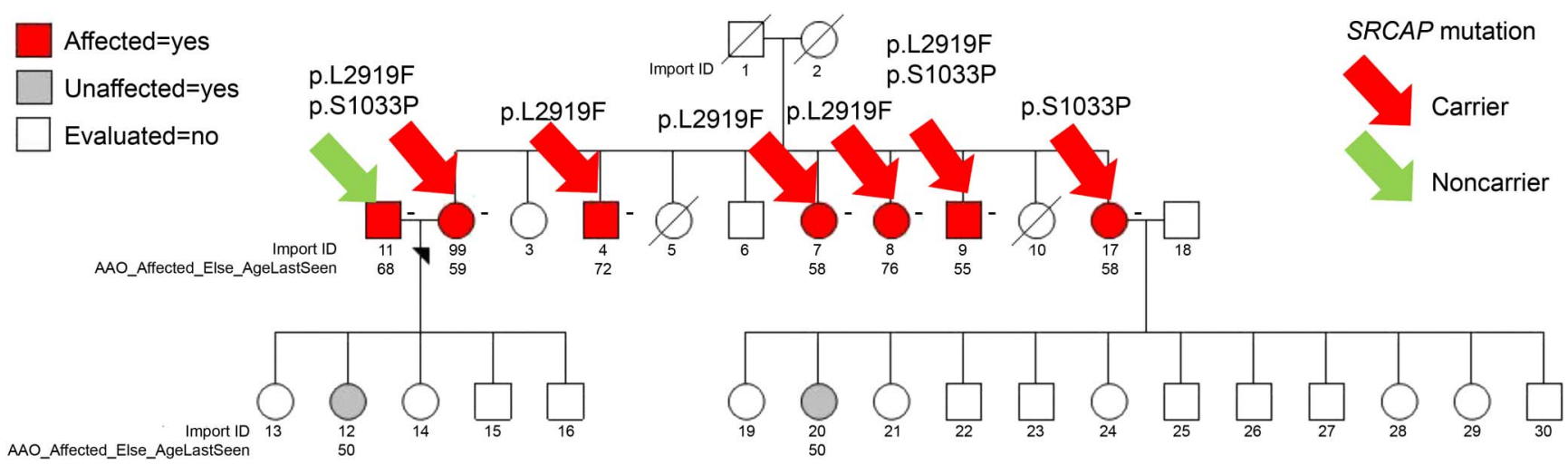

Patients 99 and 9 are compound heterozygotes with ages at onset of 59 and 55 years, respectively. Four other affected siblings who were heterozygous ( 3 p.L2919F and 1 p.S1033P) for SRCAP mutation had ages at onset of 58, 58, 72, and 76 years, respectively. Import ID: Subject ID; AAO_Affected_Else_AgeLast_Seen: Age at onset of LOAD or age at last examination for healthy individuals. LOAD = late-onset Alzheimer disease.

To reach genome-wide significance $(p=2.5 \mathrm{e}-06)$ in a gene-based test, assuming an OR of 2.0 and $5 \%$ causal SNPs and $\beta=0.8$, a gene of average size, such as $S R C A P$, requires an extremely large sample size $(\mathrm{N}=42,000)$. Therefore, we compared the combined genotyped data mentioned above with data from the ExAC database. ${ }^{24}$ Using a Fisher exact test, we found no statistically significant differences in the allele frequencies of the SRCAP variants in healthy Caribbean Hispanic controls compared with the Latino, African American, and white, non-Hispanics in the ExAC database. We then compared the frequencies of $S R C A P$ variants of LOAD patients with Caribbean Hispanic controls alone or with ExAC Latino controls, ExAC African American controls, or ExAC white, non-Hispanic controls. Using one mutation carrier per family, we compared the number of variant alleles in the LOAD patients with the number of alleles in the ExAC database. Two variants, p.P2741R and p.V2764G, were not found in the ExAC database (table 1). We used the total number of alleles genotyped or sequenced for the 10 mutations as the denominator for the Fisher exact test. The enrichment of SRCAP variants in the Caribbean Hispanic patients with LOAD was highly significant at $p=$ 1.19e-16. Similarly, the enrichment was statistically significant for white, non-Hispanics, and the African American ethnic groups in the ExAC database (table $\mathrm{e}-4 ; p=1.51 \mathrm{e}-21$ for white, non-Hispanics; $p=3 \mathrm{e}-$ 04 for African Americans). Five of the 10 SRCAP mutations were not found in the Caribbean Hispanic controls, and 4 of these were absent in the ExAC Latino data (table 1). Restricting analyses to these 5 SNPs only, the enrichment remained significant $p=3.55 \mathrm{e}-07$.

$S R C A P$ is highly intolerant toward loss of function and missense mutations. ${ }^{24,30}$ SRCAP's intolerance score (2.23) in the ExAC ranks in the 18th percentile and in the top 0.15 and 0.3 percentiles of genes ranked by the Residual Variation Intolerance Score (RVIS) using the ExAC and Exome Sequencing Project data sets, respectively.

Replication in the AD Neuroimaging Initiative data set. Using data from the Alzheimer Disease Neuroimaging Initiative (ADNI) data set, ${ }^{31}$ we repeated the analysis of the exonic regions of SRCAP. The analyses included 213 patients with LOAD, 304 individuals with mild cognitive impairment (MCI), and 214 healthy controls of white, non-Hispanic ancestry. We found a higher frequency of nonsynonymous mutations in LOAD and MCI cases (10.4\%) vs controls (7.4\%) representing a $40 \%$ enrichment. Because of the sample size, we once again compared the frequency of nonsynonymous mutations in LOAD with the ExAC database. Of interest, there was a higher frequency in LOAD vs ExAC controls $(\mathrm{OR}=1.78, p=2.3 \mathrm{e}-04)$.

SRCAP gene expression. Among the ROS-MAP cohorts, 218 of $541(40.3 \%)$ individuals were diagnosed with LOAD at death, and compared with nondemented persons, SRCAP expression was significantly higher $(\mathrm{OR}=4.9[2.1-11.6], p=0.0002)$ (figure 3A). Individuals with higher SRCAP expression also had lower cognitive scores prior to death $(\beta=-0.96, p<0.0001)$ (figure 3B). Similar associations were observed in episodic, semantic and working memory, and perceptual speed (table e-5). Using the clinical diagnosis, higher SRCAP expression was associated with greater likelihood of meeting the NIA-Reagan criteria for $\mathrm{AD}(\mathrm{OR}=4.0$ [1.7-9.2], $p=0.0015$ ) (figure 3C). Individuals with higher SRCAP expression had a greater burden of pathology $(\beta=0.3, p<0.0001)$ (figure 3D). We also accessed whole-genome sequencing data from 63 participants with brain expression. Two of 69 individuals 
Figure 3 Association of expression level of SRCAP in autopsy brain tissue with (A) AD clinical diagnoses, (B) cognitive performance, (C) AD pathologic diagnoses, and (D) global AD pathology
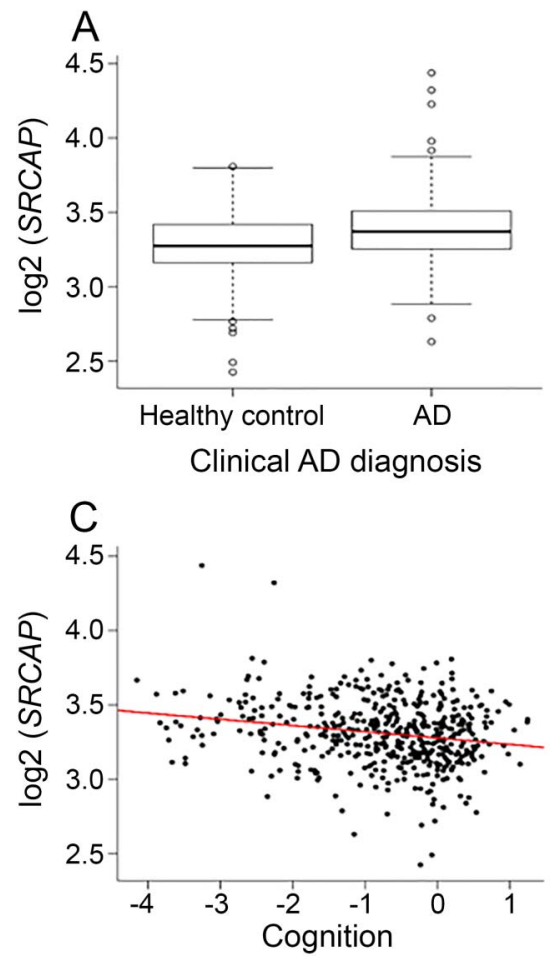

$A D=$ Alzheimer disease.
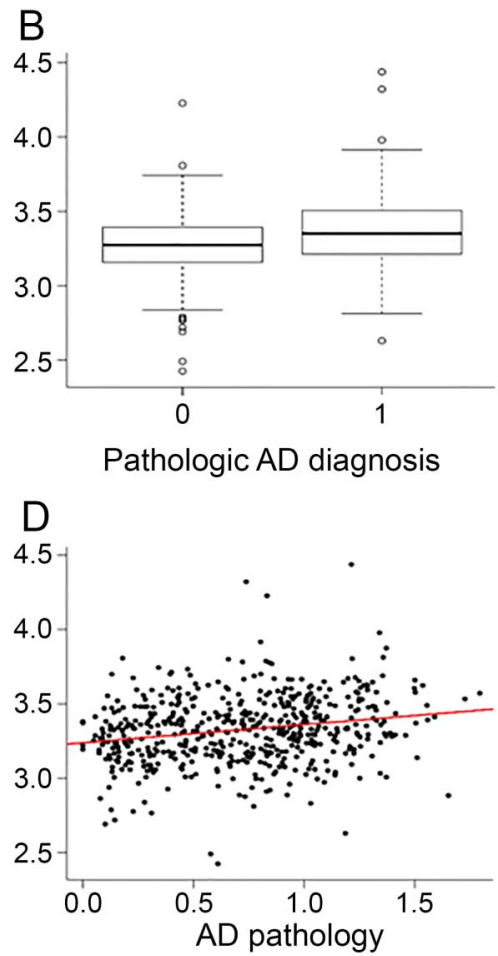

Figure 4 Comparison of SRCAP mRNA expression in affected mutation carriers, affected noncarriers, and controls

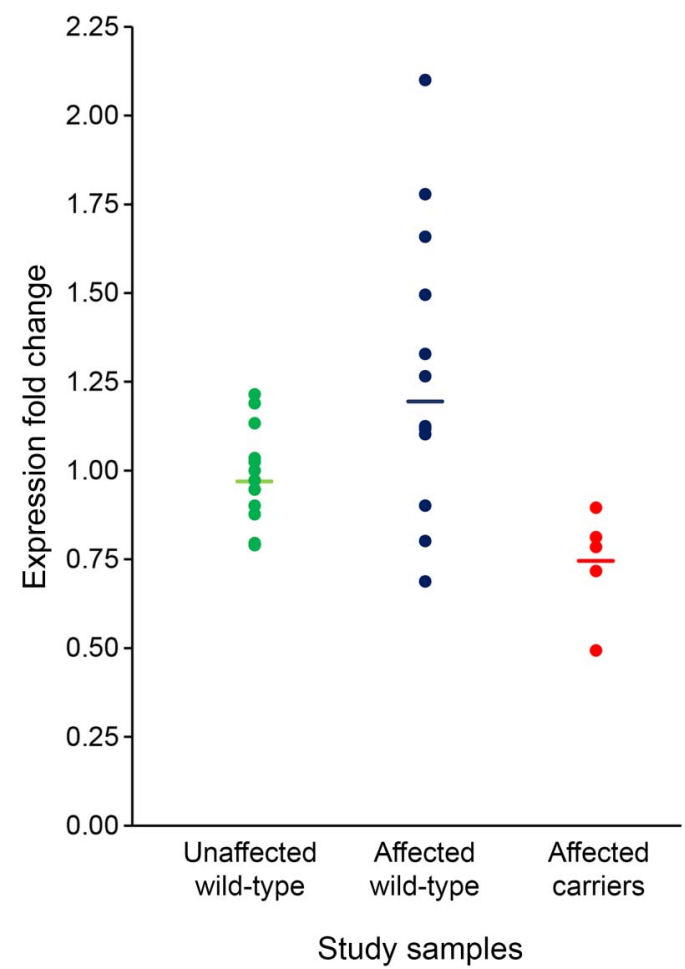

mRNA = messenger RNA.

sequenced carried 1 of the 10 SRCAP missense mutations (S1033P and L2919F), and both were diagnosed with mild cognitive impairment.

Mutation-specific gene expression. Relative messenger RNA (mRNA) expression of SRCAP (supplementary methods) in whole blood was measured in 5 mutation carriers with LOAD, 12 individuals without mutations with LOAD, and 12 elderly controls without dementia, all unrelated. After normalization against GAPDH, SRCAP mRNA expression was significantly lower in the mutation carriers with LOAD than in the other 2 groups (figure 4) (LOAD carriers 0.74, LOAD noncarriers 1.128, and controls $0.99, p=$ $0.004)$. We compared the levels of exogenous mature wild-type SRCAP mRNA in HEK293 cells to the one carrying an ultra-rare mutation (p.P2741R; minor allele frequency $=0.00128$ in Caribbean Hispanic cases and absent in ExAC). mRNA expression of mutant SRCAP (p.P2741R) was downregulated by $40 \%$ relative to wild-type SRCAP.

DISCUSSION A statistically significant enrichment of SRCAP ultra-rare mutations was found among patients within Caribbean Hispanic families multiply affected by LOAD. These mutations were observed to segregate imperfectly with disease consistent with the presence of phenocopies in large LOAD families.
Reduced mRNA expression of SRCAP was found in whole blood of mutation carriers compared with noncarriers and controls. Higher SRCAP expression was expressed in noncarriers in both blood of Caribbean Hispanic patients with LOAD and brains of Caucasian patients with LOAD, irrespective of differences in ancestry. Pathologic evidence indicates that SRCAP expression is altered in LOAD with or without mutations, correlating with characteristic clinical manifestations. Taken together, the combined data implicate a putative role for SRCAP in LOAD.

Snf2-related cyclic AMP-responsive elementbinding protein $(S R C A P)$ binds to the CREB-binding protein (CBP), which influences the transcription of CREB. CREB and the related transcription factors are involved in memory retention ${ }^{32}$ and consolidation by hippocampal neurogenesis. ${ }^{33}$ In $\mathrm{AD}$-transgenic mice and in humans with LOAD, CREB and CBP are decreased or disrupted by the accumulation of amyloid- $\beta .{ }^{34-36}$ Reduced levels have also been observed for pCREB, CBP, and related CREB coactivators $\mathrm{p} 300$ and CAMP-dependent protein kinase in the human $\mathrm{AD}$ brain and blood compared with elderly controls. ${ }^{37} \mathrm{We}$ also observed reduced mRNA expression of SRCAP in mutation carriers compared 
with healthy elderly controls and unrelated, noncarriers with $\mathrm{LOAD}$, and a reduction in SRCAP expression in cell lines transfected with SRCAP mutations. We reported an association between episodic memory and SNP rs2526690 in $C B P$ as well as nominal significance for common variants in CREBI and RBAP48 in the CREB pathway. ${ }^{38}$

$S R C A P$ regulates the CREB pathway by catalyzing H2A.Z into chromatin, which is required for gene expression and interactions with co-activators of $\mathrm{CREB}$ such as CBP. ${ }^{39,40}$ In the absence of mutations, the increased mRNA expression of $S R C A P$ in postmortem LOAD brain suggests a compensatory response to promote $C R E B$ activation in the presence of $A \beta$ accumulation. Paradoxically, in persons with $S R C A P$ mutations, disruption of the binding of SRCAP to CBP would decrease CREB-mediated transcription in brain over the lifetime. CREB may have neuroprotective qualities against $A \beta$ toxicity, and persistent downregulation of CREB resulting from decreased SRCAP could promote neurodegeneration. ${ }^{41}$ This suggests that $S R C A P$ has a regulatory role in the CREB pathway in $\mathrm{LOAD}$, regardless of the presence or absence of $S R C A P$ mutations. Ample evidence indicates disruption of CREB transcription by $A \beta$, but the effects of these ultra-rare mutations on protein function and the LOAD phenotype will need to be fully elucidated.

Truncating mutations in SRCAP cause FloatingHarbor syndrome ${ }^{42}$ a childhood disorder characterized by short stature, delayed speech, and facial abnormalities. The majority of SRCAP mutations reduced expression and create a nonfunctioning protein. Mutations in CBP, the substrate of SRCAP activation, cause a developmental disorder similar to FloatingHarbor syndrome and Rubinstein-Taybi syndrome.

This study does have limitations. We found a modest increase in the risk of LOAD among carriers of these ultra-rare SRCAP mutations in Caribbean Hispanic families, which makes replication difficult. Validation and replication efforts in other ethnic backgrounds would be essential to generalize the findings.

\section{AUTHOR CONTRIBUTIONS}

Conception and design of the study: B.N.V., G.T., S.B., R. Lefort, D.A.B., P.L.D.J., E.R., P.S.G.-H., and R.M. Acquisition and analysis of data: B.N.V., R. Lefort, P.L.N., D.R.-D., J.H.L., R.C., L.Y., D.A.B., P.L.D.J., M.M., R. Lantigua, and R.M. Drafting the manuscript or figures: B.N.V., G.T., E.R., P.S.G.-H., and R.M.

\section{STUDY FUNDING}

Supported by grants from the National Institute on Aging and the NIH, RF1AG015473, R01AG037212, P50AG008702 (R.M.), P30AG10161, RF1AG1518, R01AG17917, R01AG36936, and U01AG46152 (D.A.B. and P.L.D.J.)

\section{DISCLOSURE}

B.N. Vardarajan has served on the scientific advisory board of the Immuneering Corporation. G. Tosto has received research support from NIH/NIA, Columbia University Alzheimer's Disease Research Center
(ADRC), and the Department of Defense. R. Lefort has received research support from Columbia University Alzheimer's Disease Research Center (ADRC) and the Alzheimer's Association. L. Yu has received research support from NIH/NIA. D.A. Bennett has served on the scientific advisory boards of Vigorous Minds, Takeda Pharmaceuticals, and AbbVie; has served on the editorial boards of Neurology, Current Alzheimer Research, and Neuroepidemiology; and has received research support from NIH. P.L. De Jager has served on the scientific advisory boards of TEVA Neuroscience, Sanofi/Genzyme, and Celgene; has received speaker honoraria from Biogen Idec, Source Healthcare Analytics, Pfizer Inc., and TEVA; has served on the editorial boards of the Journal of Neuroimmunology, Neuroepigenetics, and Multiple Sclerosis; and has received research support from Biogen, Eisai, UCB, Pfizer, Sanofi/Genzyme, NIH, and the National MS Society. S. Barral and D. Reyes-Dumeyer report no disclosures. P.L. Nagy has been an employee of MNG Laboratories. J.H. Lee has received research support from NIH/NIA, NIH/NCATS, and BrightFocus Foundation. R. Cheng, M. Medrano, and R. Lantigue report no disclosures. E. Rogaeva has received research support from the Ontario Research Fund and the W. Garfield Weston Foundation. P. St George-Hyslop has received research support from the Canadian Institute of Health Research, Medical Research Council, and Wellcome Trust. R. Mayeux has received research support from NIH. Go to Neurology.org/ng for full disclosure forms.

Received February 23, 2017. Accepted in final form June 29, 2017.

\section{REFERENCES}

1. Lambert JC, Ibrahim-Verbaas CA, Harold D, et al. Metaanalysis of 74,046 individuals identifies 11 new susceptibility loci for Alzheimer's disease. Nat Genet 2013;45: 1452-1458.

2. Jonsson T, Stefansson H, Steinberg S, et al. Variant of TREM2 associated with the risk of Alzheimer's disease. N Engl J Med 2013;368:107-116.

3. Guerreiro R, Wojtas A, Bras J, et al. TREM2 variants in Alzheimer's disease. N Engl J Med 2013;368:117-127.

4. Vardarajan BN, Faber KM, Bird TD, et al. Age-specific incidence rates for dementia and Alzheimer disease in NIA-LOAD/NCRAD and EFIGA families: National Institute on Aging Genetics Initiative for Late-Onset Alzheimer Disease/National Cell Repository for Alzheimer Disease (NIA-LOAD/NCRAD) and Estudio Familiar de Influencia Genetica en Alzheimer (EFIGA). JAMA Neurol 2014;71:315-323.

5. Vardarajan BN, Schaid DJ, Reitz C, et al. Inbreeding among Caribbean Hispanics from the Dominican Republic and its effects on risk of Alzheimer disease. Genet Med 2015;17:639-643.

6. Lee JH, Cheng R, Barral S, et al. Identification of novel loci for Alzheimer disease and replication of CLU, PICALM, and BIN1 in Caribbean Hispanic individuals. Arch Neurol 2011;68:320-328.

7. McKhann G, Drachman D, Folstein M, Katzman R, Price D, Stadlan EM. Clinical diagnosis of Alzheimer's disease: report of the NINCDS-ADRDA Work group under the auspices of Department of Health and Human Services Task Force on Alzheimer's disease. Neurology 1984;34:939-944.

8. Bennett DA, Schneider JA, Aggarwal NT, et al. Decision rules guiding the clinical diagnosis of Alzheimer's disease in two community-based cohort studies compared to standard practice in a clinic-based cohort study. Neuroepidemiology 2006;27:169-176.

9. Bennett DA, Schneider JA, Arvanitakis Z, Wilson RS. Overview and findings from the Religious orders study. Curr Alzheimer Res 2012;9:628-645.

10. Bennett DA, Schneider JA, Buchman AS, Barnes LL, Boyle PA, Wilson RS. Overview and findings from the 
rush memory and aging project. Curr Alzheimer Res 2012; 9:646-663.

11. Wilson RS, Beckett LA, Barnes LL, et al. Individual differences in rates of change in cognitive abilities of older persons. Psychol Aging 2002;17:179-193.

12. Wilson RS, Boyle PA, Yu L, Segawa E, Sytsma J, Bennett DA. Conscientiousness, dementia related pathology, and trajectories of cognitive aging. Psychol Aging 2015;30: 74-82.

13. Wilson R, Barnes L, Bennett D. Assessment of lifetime participation in cognitively stimulating activities. J Clin Exp Neuropsychol 2003;25:634-642.

14. Schneider JA, Arvanitakis Z, Bang W, Bennett DA. Mixed brain pathologies account for most dementia cases in community-dwelling older persons. Neurology 2007;69: 2197-2204.

15. Bennett DA, Wilson RS, Schneider JA, et al. Apolipoprotein $\mathrm{E}$ epsilon4 allele, $\mathrm{AD}$ pathology, and the clinical expression of Alzheimer's disease. Neurology 2003;60: 246-252.

16. Schneider JA, Arvanitakis Z, Leurgans SE, Bennett DA. The neuropathology of probable Alzheimer disease and mild cognitive impairment. Ann Neurol 2009;66: 200-208.

17. Hyman BT, Trojanowski JQ. Consensus recommendations for the postmortem diagnosis of Alzheimer disease from the National Institute on Aging and the Reagan Institute Working Group on diagnostic criteria for the neuropathological assessment of Alzheimer disease. J Neuropathol Exp Neurol 1997;56:1095-1097.

18. Abrams KR, Gillies CL, Lambert PC. Meta-analysis of heterogeneously reported trials assessing change from baseline. Stat Med 2005;24:3823-3844.

19. Li H, Durbin R. Fast and accurate short read alignment with Burrows-Wheeler transform. Bioinformatics 2009; 25:1754-1760.

20. McKenna A, Hanna M, Banks E, et al. The Genome Analysis Toolkit: a MapReduce framework for analyzing next-generation DNA sequencing data. Genome Res 2010;20:1297-1303.

21. Wang K, Li M, Hakonarson H. ANNOVAR: functional annotation of genetic variants from high-throughput sequencing data. Nucleic Acids Res 2010;38:e164.

22. Kumar P, Henikoff S, Ng PC. Predicting the effects of coding non-synonymous variants on protein function using the SIFT algorithm. Nat Protoc 2009;4:1073-1081.

23. Adzhubei IA, Schmidt S, Peshkin L, et al. A method and server for predicting damaging missense mutations. Nat Methods 2010;7:248-249.

24. Lek M, Karczewski KJ, Minikel EV, et al. Analysis of protein-coding genetic variation in 60,706 humans. Nature 2016;536:285-291.

25. Levin JZ, Yassour M, Adiconis X, et al. Comprehensive comparative analysis of strand-specific RNA sequencing methods. Nat Methods 2010;7:709-715.

26. Adiconis X, Borges-Rivera D, Satija R, et al. Comparative analysis of RNA sequencing methods for degraded or lowinput samples. Nat Methods 2013;10:623-629.

27. Johnson WE, Li C, Rabinovic A. Adjusting batch effects in microarray expression data using empirical Bayes methods. Biostatistics 2007;8:118-127.
28. Adzhubei I, Jordan DM, Sunyaev SR. Predicting functional effect of human missense mutations using PolyPhen-2. Curr Protoc Hum Genet 2013; Chap 7. Unit7.20.

29. Kircher M, Witten DM, Jain P, O'Roak BJ, Cooper GM, Shendure J. A general framework for estimating the relative pathogenicity of human genetic variants. Nat Genet 2014;46:310-315.

30. Petrovski S, Wang Q, Heinzen EL, Allen AS, Goldstein DB. Genic intolerance to functional variation and the interpretation of personal genomes. PLoS Genet 2013;9: e1003709.

31. Saykin AJ, Shen L, Foroud TM, et al. Alzheimer's Disease Neuroimaging Initiative biomarkers as quantitative phenotypes: genetics core aims, progress, and plans. Alzheimers Dement 2010;6:265-273.

32. Lakhina V, Arey RN, Kaletsky R, et al. Genome-wide functional analysis of CREB/long-term memory-dependent transcription reveals distinct basal and memory gene expression programs. Neuron 2015;85:330-345.

33. Ortega-Martinez S. A new perspective on the role of the CREB family of transcription factors in memory consolidation via adult hippocampal neurogenesis. Front $\mathrm{Mol}$ Neurosci 2015;8:46.

34. Duclot F, Meffre J, Jacquet C, Gongora C, Maurice T. Mice knock out for the histone acetyltransferase p300/CREB binding protein-associated factor develop a resistance to amyloid toxicity. Neuroscience 2010;167: 850-863.

35. Pugazhenthi S, Wang M, Pham S, Sze CI, Eckman CB. Downregulation of CREB expression in Alzheimer's brain and in Abeta-treated rat hippocampal neurons. Mol Neurodegener 2011;6:60.

36. Satoh J, Tabunoki H, Arima K. Molecular network analysis suggests aberrant CREB-mediated gene regulation in the Alzheimer disease hippocampus. Dis Markers 2009; 27:239-252.

37. Bartolotti N, Bennett DA, Lazarov O. Reduced pCREB in Alzheimer's disease prefrontal cortex is reflected in peripheral blood mononuclear cells. Mol Psychiatry 2016;21: 1158-1166.

38. Barral S, Reitz C, Small SA, Mayeux R. Genetic variants in a "cAMP element binding protein" (CREB)-dependent histone acetylation pathway influence memory performance in cognitively healthy elderly individuals. Neurobiol Aging 2014;35:2881.e7-2881.e10.

39. Wong MM, Cox LK, Chrivia JC. The chromatin remodeling protein, SRCAP, is critical for deposition of the histone variant H2A.Z at promoters. J Biol Chem 2007;282: 26132-26139.

40. Zovkic IB, Paulukaitis BS, Day JJ, Etikala DM, Sweatt JD. Histone H2A.Z subunit exchange controls consolidation of recent and remote memory. Nature 2014;515: 582-586.

41. Monroy MA, Ruhl DD, Xu X, Granner DK, Yaciuk P, Chrivia JC. Regulation of cAMP-responsive element-binding protein-mediated transcription by the SNF2/SWI-related protein, SRCAP. J Biol Chem 2001;276:40721-40726.

42. Nikkel SM, Dauber A, de Munnik S, et al. The phenotype of Floating-Harbor syndrome: clinical characterization of 52 individuals with mutations in exon 34 of SRCAP. Orphanet J Rare Dis 2013;8:63. 


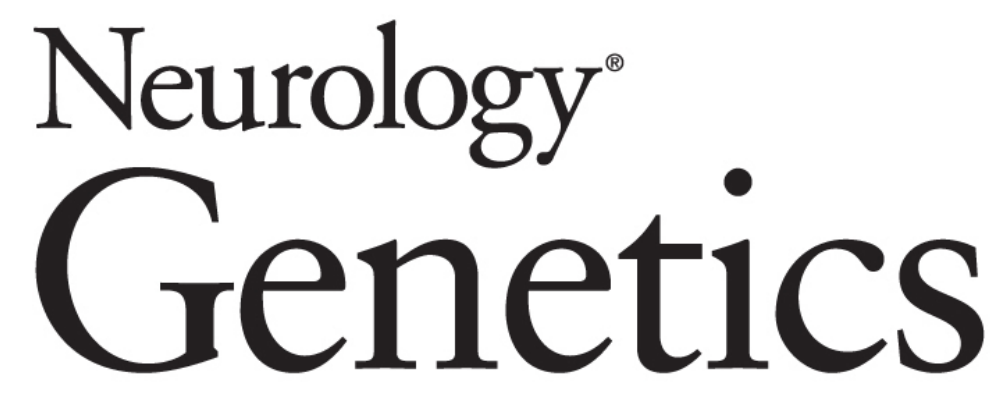

Ultra-rare mutations in SRCAP segregate in Caribbean Hispanic families with Alzheimer disease

Badri N. Vardarajan, Giuseppe Tosto, Roger Lefort, et al. Neurol Genet 2017;3;

DOI 10.1212/NXG.0000000000000178

This information is current as of August 24, 2017

Neurol Genet is an official journal of the American Academy of Neurology. Published since April 2015, it is an open-access, online-only, continuous publication journal. Copyright Copyright ( 2017 The Author(s). Published by Wolters Kluwer Health, Inc. on behalf of the American Academy of Neurology.. All rights reserved. Online ISSN: 2376-7839.

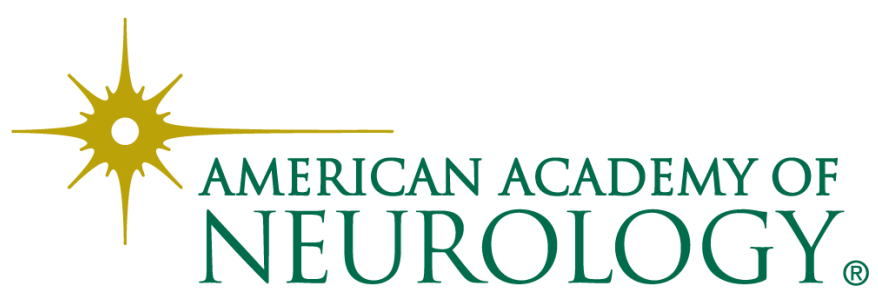




\section{Updated Information \& Services}

\section{Supplementary Material}

References

Subspecialty Collections

Permissions \& Licensing

Reprints including high resolution figures, can be found at: http://ng.neurology.org/content/3/5/e178.full.html

Supplementary material can be found at: http://ng.neurology.org/content/suppl/2017/08/24/3.5.e178.DC1

This article cites 41 articles, 3 of which you can access for free at: http://ng.neurology.org/content/3/5/e178.full.html\#\#ref-list-1

This article, along with others on similar topics, appears in the following collection(s):

\section{All Genetics}

http://ng.neurology.org//cgi/collection/all_genetics

Alzheimer's disease

http://ng.neurology.org//cgi/collection/alzheimers_disease

Association studies in genetics

http://ng.neurology.org//cgi/collection/association_studies_in_genetics

Information about reproducing this article in parts (figures,tables) or in its entirety can be found online at:

http://ng.neurology.org/misc/about.xhtml\#permissions

Information about ordering reprints can be found online:

http://ng.neurology.org/misc/addir.xhtml\#reprintsus

Neurol Genet is an official journal of the American Academy of Neurology. Published since April 2015, it is an open-access, online-only, continuous publication journal. Copyright Copyright $\odot 2017$ The Author(s). Published by Wolters Kluwer Health, Inc. on behalf of the American Academy of Neurology.. All rights reserved. Online ISSN: 2376-7839.

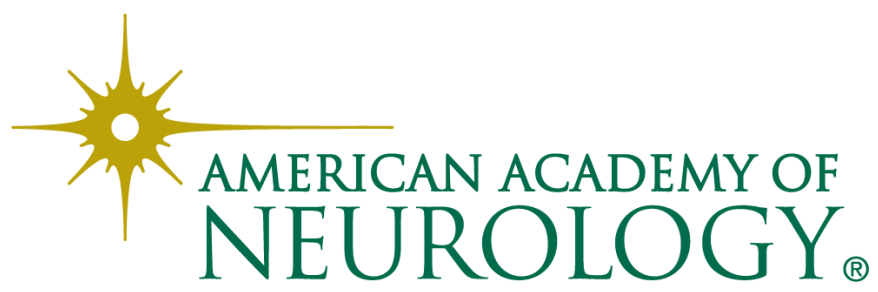

